\title{
Strong convergence theorems by hybrid methods for the split common null point problem in Banach spaces
}

\author{
Wataru Takahashi ${ }^{1,2,3}$ and Jen-Chih Yao ${ }^{4, *^{*}}$
}

\author{
"Correspondence: \\ yaojc@mail.cmu.edu.tw \\ ${ }^{4}$ Center for General Education, \\ China Medical University, Taichung, \\ 40402, Taiwan \\ ${ }^{5}$ Department of Mathematics, King \\ Abdulaziz University, P.O. Box 80203 \\ Jeddah, 21589, Saudi Arabia \\ Full list of author information is \\ available at the end of the article
}

\begin{abstract}
In this paper, we consider the split common null point problem in Banach spaces. Then using the hybrid method and the shrinking projection method in mathematical programming, we prove strong convergence theorems for finding a solution of the split common null point problem in Banach spaces.
\end{abstract}

MSC: $47 \mathrm{H} 05 ; 47 \mathrm{HO}$

Keywords: split common null point problem; fixed point; metric resolvent; hybrid method; shrinking projection method; duality mapping

\section{Introduction}

Let $H_{1}$ and $H_{2}$ be two real Hilbert spaces. Let $D$ and $Q$ be nonempty, closed, and convex subsets of $H_{1}$ and $H_{2}$, respectively. Let $A: H_{1} \rightarrow H_{2}$ be a bounded linear operator. Then the split feasibility problem [1] is to find $z \in H_{1}$ such that $z \in D \cap A^{-1} Q$. Defining $U=A^{*}\left(I-P_{Q}\right) A$ in the split feasibility problem, we see that $U: H_{1} \rightarrow H_{1}$ is an inverse strongly monotone operator [2], where $A^{*}$ is the adjoint operator of $A$ and $P_{Q}$ is the metric projection of $H_{2}$ onto $Q$. Furthermore, if $D \cap A^{-1} Q$ is nonempty, then $z \in D \cap A^{-1} Q$ is equivalent to

$$
z=P_{D}\left(I-\lambda A^{*}\left(I-P_{Q}\right) A\right) z,
$$

where $\lambda>0$ and $P_{D}$ is the metric projection of $H_{1}$ onto $D$. Using such results regarding nonlinear operators and fixed points, many authors have studied the split feasibility problem in Hilbert spaces; see, for instance, [2-6]. Recently, Takahashi [7] and [8] extended such an equivalent relation (1.1) in Hilbert spaces to Banach spaces and then obtained strong convergence theorems for finding a solution of the split feasibility problem in Banach spaces. Very recently, using the hybrid method by Nakajo and Takahashi [9] in mathematical programming, Alsulami et al. [10] prove strong convergence theorems for finding a solution of the split feasibility problem in Banach spaces; see also [11, 12].

Theorem 1 ([10]) Let $H$ be a Hilbert space and let $F$ be a strictly convex, reflexive and smooth Banach space. Let $J_{F}$ be the duality mapping on F. Let $C$ and D be nonempty, closed,

(C) 2015 Takahashi and Yao. This article is distributed under the terms of the Creative Commons Attribution 4.0 International License (http://creativecommons.org/licenses/by/4.0/), which permits unrestricted use, distribution, and reproduction in any medium, provided you give appropriate credit to the original author(s) and the source, provide a link to the Creative Commons license, and indicate if changes were made. 
and convex subsets of $H$ and $F$, respectively. Let $P_{C}$ and $P_{D}$ be the metric projections of $H$ onto $C$ and $F$ onto $D$, respectively. Let $A: H \rightarrow F$ be a bounded linear operator such that $A \neq 0$ and let $A^{*}$ be the adjoint operator of $A$. Suppose that $C \cap A^{-1} D \neq \emptyset$. Let $x_{1} \in H$ and let $\left\{x_{n}\right\}$ be a sequence generated by

$$
\left\{\begin{array}{l}
z_{n}=P_{C}\left(x_{n}-r A^{*} J_{F}\left(A x_{n}-P_{D} A x_{n}\right)\right), \\
y_{n}=\alpha_{n} x_{n}+\left(1-\alpha_{n}\right) z_{n}, \\
C_{n}=\left\{z \in H:\left\|y_{n}-z\right\| \leq\left\|x_{n}-z\right\|\right\}, \\
Q_{n}=\left\{z \in H:\left\langle x_{n}-z, x_{1}-x_{n}\right\rangle \geq 0\right\}, \\
x_{n+1}=P_{C_{n} \cap Q_{n}} x_{1}, \quad \forall n \in \mathbb{N},
\end{array}\right.
$$

where $0 \leq \alpha_{n} \leq a<1$ for some $a \in \mathbb{R}$ and $0<r\|A\|^{2}<2$. Then $\left\{x_{n}\right\}$ converges strongly to a point $z_{0} \in C \cap A^{-1} D$, where $z_{0}=P_{C \cap A^{-1} D^{x_{1}}}$.

Takahashi [8] also obtained the following result from the idea of the shrinking projection method by Takahashi et al. [13].

Theorem 2 ([8]) Let $H$ be a Hilbert space and let $F$ be a uniformly convex Banach space whose norm is Fréchet differentiable. Let $J_{F}$ be the duality mapping on F. Let $C$ and $D$ be nonempty, closed, and convex subsets of $H$ and $F$, respectively. Let $P_{C}$ and $P_{D}$ be the metric projections of $H$ onto $C$ and $F$ onto $D$, respectively. Let $A: H \rightarrow F$ be a bounded linear operator such that $A \neq 0$ and let $A^{*}$ be the adjoint operator of $A$. Suppose that $C \cap A^{-1} D \neq \emptyset$. Let $\left\{u_{n}\right\}$ be a sequence in $H$ such that $u_{n} \rightarrow u$. Let $x_{1} \in H, C_{1}=H$, and $\left\{x_{n}\right\}$ be a sequence generated by

$$
\left\{\begin{array}{l}
z_{n}=P_{C}\left(x_{n}-r A^{*} J_{F}\left(A x_{n}-P_{D} A x_{n}\right)\right), \\
C_{n+1}=\left\{z \in H:\left\|z_{n}-z\right\| \leq\left\|x_{n}-z\right\|\right\} \cap C_{n}, \\
x_{n+1}=P_{C_{n+1}} u_{n+1}, \quad \forall n \in \mathbb{N},
\end{array}\right.
$$

where $0<r\|A\|^{2} \leq 2$. Then $\left\{x_{n}\right\}$ converges strongly to a point $z_{0} \in C \cap A^{-1} D$, where $z_{0}=$ $P_{C \cap A^{-1} D} u$.

On the other hand, Byrne et al. [3] considered the following problem: Given set-valued mappings $A_{i}: H_{1} \rightarrow 2^{H_{1}}, 1 \leq i \leq m$, and $B_{j}: H_{2} \rightarrow 2^{H_{2}}, 1 \leq j \leq n$, respectively, and bounded linear operators $T_{j}: H_{1} \rightarrow H_{2}, 1 \leq j \leq n$, the split common null point problem [3] is to find a point $z \in H_{1}$ such that

$$
z \in\left(\bigcap_{i=1}^{m} A_{i}^{-1} 0\right) \cap\left(\bigcap_{j=1}^{n} T_{j}^{-1}\left(B_{j}^{-1} 0\right)\right),
$$

where $A_{i}^{-1} 0$ and $B_{j}^{-1} 0$ are null point sets of $A_{i}$ and $B_{j}$, respectively.

In this paper, motivated by these problems and results for the problems in Hilbert spaces, we consider the split common null point problem in Banach spaces. Then using the hybrid method and the shrinking projection method in mathematical programming, we prove two strong convergence theorems for finding a solution of the split common null point problem in Banach spaces. 


\section{Preliminaries}

Throughout this paper, we denote by $\mathbb{N}$ the set of positive integers and by $\mathbb{R}$ the set of real numbers. Let $H$ be a real Hilbert space with inner product $\langle\cdot, \cdot\rangle$ and norm $\|\cdot\|$, respectively. For $x, y \in H$ and $\lambda \in \mathbb{R}$, we have from [14]

$$
\begin{aligned}
& \|x+y\|^{2} \leq\|x\|^{2}+2\langle y, x+y\rangle ; \\
& \|\lambda x+(1-\lambda) y\|^{2}=\lambda\|x\|^{2}+(1-\lambda)\|y\|^{2}-\lambda(1-\lambda)\|x-y\|^{2} .
\end{aligned}
$$

Furthermore we see that, for $x, y, u, v \in H$,

$$
2\langle x-y, u-v\rangle=\|x-v\|^{2}+\|y-u\|^{2}-\|x-u\|^{2}-\|y-v\|^{2} .
$$

Let $C$ be a nonempty, closed, and convex subset of a Hilbert space $H$. The nearest point projection of $H$ onto $C$ is denoted by $P_{C}$, that is, $\left\|x-P_{C} x\right\| \leq\|x-y\|$ for all $x \in H$ and $y \in C$. Such $P_{C}$ is called the metric projection of $H$ onto $C$. We know that the metric projection $P_{C}$ is firmly nonexpansive, i.e.,

$$
\left\|P_{C} x-P_{C} y\right\|^{2} \leq\left\langle P_{C} x-P_{C} y, x-y\right\rangle
$$

for all $x, y \in H$. Furthermore $\left\langle x-P_{C} x, y-P_{C} x\right\rangle \leq 0$ holds for all $x \in H$ and $y \in C$; see [15].

Let $E$ be a real Banach space with norm $\|\cdot\|$ and let $E^{*}$ be the dual space of $E$. We denote the value of $y^{*} \in E^{*}$ at $x \in E$ by $\left\langle x, y^{*}\right\rangle$. When $\left\{x_{n}\right\}$ is a sequence in $E$, we denote the strong convergence of $\left\{x_{n}\right\}$ to $x \in E$ by $x_{n} \rightarrow x$ and the weak convergence by $x_{n} \rightarrow x$. The modulus $\delta$ of convexity of $E$ is defined by

$$
\delta(\epsilon)=\inf \left\{1-\frac{\|x+y\|}{2}:\|x\| \leq 1,\|y\| \leq 1,\|x-y\| \geq \epsilon\right\}
$$

for every $\epsilon$ with $0 \leq \epsilon \leq 2$. A Banach space $E$ is said to be uniformly convex if $\delta(\epsilon)>0$ for every $\epsilon>0$. It is well known that a Banach space $E$ is uniformly convex if and only if for any two sequences $\left\{x_{n}\right\}$ and $\left\{y_{n}\right\}$ in $E$ such that

$$
\lim _{n \rightarrow \infty}\left\|x_{n}\right\|=\lim _{n \rightarrow \infty}\left\|y_{n}\right\|=1 \quad \text { and } \quad \lim _{n \rightarrow \infty}\left\|x_{n}+y_{n}\right\|=2,
$$

$\lim _{n \rightarrow \infty}\left\|x_{n}-y_{n}\right\|=0$ holds. A uniformly convex Banach space is strictly convex and reflexive. We also know that a uniformly convex Banach space has the Kadec-Klee property, i.e., $x_{n} \rightarrow u$ and $\left\|x_{n}\right\| \rightarrow\|u\|$ imply $x_{n} \rightarrow u$.

The duality mapping $J$ from $E$ into $2^{E^{*}}$ is defined by

$$
J x=\left\{x^{*} \in E^{*}:\left\langle x, x^{*}\right\rangle=\|x\|^{2}=\left\|x^{*}\right\|^{2}\right\}
$$

for every $x \in E$. Let $U=\{x \in E:\|x\|=1\}$. The norm of $E$ is said to be Gâteaux differentiable if for each $x, y \in U$, the limit

$$
\lim _{t \rightarrow 0} \frac{\|x+t y\|-\|x\|}{t}
$$


exists. In the case, $E$ is called smooth. We know that $E$ is smooth if and only if $J$ is a singlevalued mapping of $E$ into $E^{*}$. We also know that $E$ is reflexive if and only if $J$ is surjective, and $E$ is strictly convex if and only if $J$ is one-to-one. Therefore, if $E$ is a smooth, strictly convex and reflexive Banach space, then $J$ is a single-valued bijection and in this case, the inverse mapping $J^{-1}$ coincides with the duality mapping $J_{*}$ on $E^{*}$. The norm of $E$ is said to be Fréchet differentiable if for each $x \in U$, the limit (2.5) is attained uniformly for $y \in U$. It is known that if the norm of $E$ is Fréchet differentiable, then $J$ is norm to norm continuous. For more details, see [15] and [16]. We know the following result.

Lemma 3 ([15]) Let E be a smooth Banach space and let $J$ be the duality mapping on $E$. Then $\langle x-y, J x-J y\rangle \geq 0$ for all $x, y \in E$. Furthermore, if $E$ is strictly convex and $\langle x-y, J x-J y\rangle=$ 0 , then $x=y$.

Let $C$ be a nonempty, closed, and convex subset of a strictly convex and reflexive Banach space $E$. Then we know that, for any $x \in E$, there exists a unique element $z \in C$ such that $\|x-z\| \leq\|x-y\|$ for all $y \in C$. Putting $z=P_{C} x$, we call $P_{C}$ the metric projection of $E$ onto $C$.

Lemma 4 ([15]) Let E be a smooth, strictly convex, and reflexive Banach space. Let $C$ be a nonempty, closed, and convex subset of $E$ and let $x_{1} \in E$ and $z \in C$. Then the following conditions are equivalent:

(1) $z=P_{C} x_{1}$

(2) $\left\langle z-y, J\left(x_{1}-z\right)\right\rangle \geq 0, \forall y \in C$.

Let $E$ be a smooth Banach space and let $J$ be the duality mapping on $E$. Define a function $\phi: E \times E \rightarrow \mathbb{R}$ by

$$
\phi(x, y)=\|x\|^{2}-2\langle x, J y\rangle+\|y\|^{2}, \quad \forall x, y \in E .
$$

Observe that, in a Hilbert space $H, \phi(x, y)=\|x-y\|^{2}$ for all $x, y \in H$. Furthermore, we know that, for each $x, y, z, w \in E$,

$$
\begin{aligned}
& (\|x\|-\|y\|)^{2} \leq \phi(x, y) \leq(\|x\|+\|y\|)^{2} \\
& \phi(x, y)=\phi(x, z)+\phi(z, y)+2\langle x-z, J z-J y\rangle ; \\
& 2\langle x-y, J z-J w\rangle=\phi(x, w)+\phi(y, z)-\phi(x, z)-\phi(y, w) .
\end{aligned}
$$

If $E$ is additionally assumed to be strictly convex, then

$$
\phi(x, y)=0 \quad \text { if and only if } \quad x=y .
$$

The following lemma was proved by Kamimura and Takahashi [17].

Lemma 5 ([17]) Let E be a uniformly convex Banach space and let $r>0$. Then there exists a strictly increasing, continuous, and convex function $g:[0,2 r] \rightarrow[0, \infty)$ such that $g(0)=0$ and

$$
g(\|x-y\|) \leq \phi(x, y)
$$

for all $x, y \in B_{r}$, where $B_{r}=\{z \in E:\|z\| \leq r\}$. 
Let $E$ be a Banach space and let $A$ be a mapping of $E$ into $2^{E^{*}}$. The effective domain of $A$ is denoted by $\operatorname{dom}(A)$, that is, $\operatorname{dom}(A)=\{x \in E: A x \neq \emptyset\}$. A multi-valued mapping $A$ on $E$ is said to be monotone if $\left\langle x-y, u^{*}-v^{*}\right\rangle \geq 0$ for all $x, y \in \operatorname{dom}(A), u^{*} \in A x$, and $v^{*} \in A y$. A monotone operator $A$ on $E$ is said to be maximal if its graph is not properly contained in the graph of any other monotone operator on $E$. The following theorem is due to Browder [18]; see also [16], Theorem 3.5.4.

Theorem 6 ([18]) Let E be a uniformly convex and smooth Banach space and let $J$ be the duality mapping of $E$ into $E^{*}$. Let $A$ be a monotone operator of $E$ into $2^{E^{*}}$. Then $A$ is maximal if and only if for any $r>0$,

$$
R(J+r A)=E^{*},
$$

where $R(J+r A)$ is the range of $J+r A$.

Let $E$ be a uniformly convex Banach space with a Gâteaux differentiable norm and let $A$ be a maximal monotone operator of $E$ into $2^{E^{*}}$. For all $x \in E$ and $r>0$, we consider the following equation:

$$
0 \in J\left(x_{r}-x\right)+r A x_{r}
$$

This equation has a unique solution $x_{r}$. We define $J_{r}$ by $x_{r}=J_{r} x$. Such $J_{r}, r>0$ are called the metric resolvents of $A$. The set of null points of $A$ is defined by $A^{-1} 0=\{z \in E: 0 \in A z\}$. We know that $A^{-1} 0$ is closed and convex; see [16]. Let $E$ be a uniformly convex and smooth Banach space $E$ and let $J_{r}$ be the metric resolvent of $A$ for $r>0$. Using Lemma 5, we can prove that the metric resolvent $J_{r}$ is continuous. In fact, let $x_{n} \rightarrow x_{0}$. Since $J_{r}$ is the metric resolvent of $A$ for $r>0$, we have from [19]

$$
\left\langle J_{r} x_{n}-y, J\left(x_{n}-J_{r} x_{n}\right)\right\rangle \geq 0, \quad \forall y \in A^{-1} 0 .
$$

Then we have $\left\langle J_{r} x_{n}-x_{n}+x_{n}-y, J\left(x_{n}-J_{r} x_{n}\right)\right\rangle \geq 0$ and hence

$$
\begin{aligned}
\left\|x_{n}-y\right\|\left\|x_{n}-J_{r} x_{n}\right\| & \geq\left\langle x_{n}-y, J\left(x_{n}-J_{r} x_{n}\right)\right\rangle \\
& \geq\left\langle x_{n}-J_{r} x_{n}, J\left(x_{n}-J_{r} x_{n}\right)\right\rangle \\
& =\left\|x_{n}-J_{r} x_{n}\right\|^{2} .
\end{aligned}
$$

This means that $\left\{x_{n}-J_{r} x_{n}\right\}$ is bounded. Furthermore, since $J_{r}$ is the metric resolvent of $A$ for $r>0$, we know that

$$
\left\langle J_{r} x_{n}-J_{r} x_{0}, J\left(x_{n}-J_{r} x_{n}\right)-J\left(x_{0}-J_{r} x_{0}\right)\right\rangle \geq 0
$$

Using (2.8) and Lemma 5, we see that

$$
\begin{aligned}
& 2\left\langle x_{n}-x_{0}, J\left(x_{n}-J_{r} x_{n}\right)-J\left(x_{0}-J_{r} x_{0}\right)\right\rangle \\
& \quad \geq 2\left\langle x_{n}-J_{r} x_{n}-\left(x_{0}-J_{r} x_{0}\right), J\left(x_{n}-J_{r} x_{n}\right)-J\left(x_{0}-J_{r} x_{0}\right)\right\rangle
\end{aligned}
$$




$$
\begin{aligned}
& =\phi\left(x_{n}-J_{r} x_{n}, x_{0}-J_{r} x_{0}\right)+\phi\left(x_{0}-J_{r} x_{0}, x_{n}-J_{r} x_{n}\right) \\
& \geq g\left(\left\|x_{n}-J_{r} x_{n}-\left(x_{0}-J_{r} x_{0}\right)\right\|\right)+g\left(\left\|x_{0}-J_{r} x_{0}-\left(x_{n}-J_{r} x_{n}\right)\right\|\right) \\
& =2 g\left(\left\|x_{n}-J_{r} x_{n}-\left(x_{0}-J_{r} x_{0}\right)\right\|\right),
\end{aligned}
$$

where $g$ is a strictly increasing, continuous, and convex function in Lemma 5. Therefore, if $x_{n} \rightarrow x_{0}$, then $J_{r} x_{n} \rightarrow J_{r} x_{0}$. Therefore, $J_{r}$ is continuous.

Let $A$ be a maximal monotone operator on a Hilbert space $H$. In a Hilbert space $H$, the metric resolvent $J_{r}$ of $A$ is called the resolvent of $A$ simply. It is known that the resolvent $J_{r}$ of $A$ for $r>0$ is firmly nonexpansive, i.e.,

$$
\left\|J_{r} x-J_{r} y\right\|^{2} \leq\left\langle x-y, J_{r} x-J_{r} y\right\rangle, \quad \forall x, y \in H .
$$

It is also known that $\left\|J_{\lambda} x-J_{\mu} x\right\| \leq(|\lambda-\mu| / \lambda)\left\|x-J_{\lambda} x\right\|$ holds for all $\lambda, \mu>0$ and $x \in H$; see $[15,20]$ for more details. As a matter of fact, we have the following lemma due to Takahashi et al. [21].

Lemma 7 ([21]) Let $H$ be a Hilbert space and let $B$ be a maximal monotone operator on $H$. For $r>0$ and $x \in H$, define the resolvent $J_{r} x$. Then the following holds:

$$
\frac{s-t}{s}\left\langle J_{s} x-J_{t} x, J_{s} x-x\right\rangle \geq\left\|J_{s} x-J_{t} x\right\|^{2}
$$

for all $s, t>0$ and $x \in H$.

For a sequence $\left\{C_{n}\right\}$ of nonempty, closed, and convex subsets of a Banach space $E$, define s-Li ${ }_{n} C_{n}$ and $\mathrm{w}-\mathrm{Ls}_{n} C_{n}$ as follows: $x \in \mathrm{s}-\mathrm{Li}_{n} C_{n}$ if and only if there exists $\left\{x_{n}\right\} \subset E$ such that $\left\{x_{n}\right\}$ converges strongly to $x$ and $x_{n} \in C_{n}$ for all $n \in \mathbb{N}$. Similarly, $y \in \mathrm{w}-\mathrm{Ls}_{n} C_{n}$ if and only if there exist a subsequence $\left\{C_{n_{i}}\right\}$ of $\left\{C_{n}\right\}$ and a sequence $\left\{y_{i}\right\} \subset E$ such that $\left\{y_{i}\right\}$ converges weakly to $y$ and $y_{i} \in C_{n_{i}}$ for all $i \in \mathbb{N}$. If $C_{0}$ satisfies

$$
C_{0}=\mathrm{s}-\mathrm{Li}_{n} C_{n}=\mathrm{w}-\mathrm{Ls}_{n} C_{n},
$$

it is said that $\left\{C_{n}\right\}$ converges to $C_{0}$ in the sense of Mosco [22] and we write $C_{0}=$ $\mathrm{M}-\lim _{n \rightarrow \infty} C_{n}$. It is easy to show that if $\left\{C_{n}\right\}$ is nonincreasing with respect to inclusion, then $\left\{C_{n}\right\}$ converges to $\bigcap_{n=1}^{\infty} C_{n}$ in the sense of Mosco. For more details, see [22]. The following lemma was proved by Tsukada [23].

Lemma 8 ([23]) Let E be a uniformly convex Banach space. Let $\left\{C_{n}\right\}$ be a sequence of nonempty, closed, and convex subsets of $E$. If $C_{0}=\mathrm{M}-\lim _{n \rightarrow \infty} C_{n}$ exists and nonempty, then for each $x \in E,\left\{P_{C_{n}} x\right\}$ converges strongly to $P_{C_{0}} x$, where $P_{C_{n}}$ and $P_{C_{0}}$ are the metric projections of $E$ onto $C_{n}$ and $C_{0}$, respectively.

\section{Main results}

In this section, using the hybrid method in mathematical programming, we first prove a strong convergence theorem for finding a solution of the split common null point problem in Banach spaces. 
Theorem 9 Let $H$ be a Hilbert space and let $F$ be a uniformly convex and smooth Banach space. Let $J_{F}$ be the duality mapping on $F$. Let $A$ and $B$ be maximal monotone operators of $H$ into $2^{H}$ and $F$ into $2^{F^{*}}$ such that $A^{-1} 0 \neq \emptyset$ and $B^{-1} 0 \neq \emptyset$, respectively. Let $J_{\lambda}$ be the resolvent of $A$ for $\lambda>0$ and let $Q_{\mu}$ be the metric resolvent of $B$ for $\mu>0$. Let $T: H \rightarrow F$ be a bounded linear operator such that $T \neq 0$ and let $T^{*}$ be the adjoint operator of $T$. Suppose that $A^{-1} 0 \cap T^{-1}\left(B^{-1} 0\right) \neq \emptyset$. Let $x_{1} \in H$ and let $\left\{x_{n}\right\}$ be a sequence generated by

$$
\left\{\begin{array}{l}
z_{n}=J_{\lambda_{n}}\left(x_{n}-\lambda_{n} T^{*} J_{F}\left(T x_{n}-Q_{\mu_{n}} T x_{n}\right)\right), \\
y_{n}=\alpha_{n} x_{n}+\left(1-\alpha_{n}\right) z_{n}, \\
C_{n}=\left\{z \in H:\left\|y_{n}-z\right\| \leq\left\|x_{n}-z\right\|\right\}, \\
D_{n}=\left\{z \in H:\left\langle x_{n}-z, x_{1}-x_{n}\right\rangle \geq 0\right\}, \\
x_{n+1}=P_{C_{n} \cap D_{n}} x_{1}, \quad \forall n \in \mathbb{N},
\end{array}\right.
$$

where $\left\{\alpha_{n}\right\} \subset[0,1]$ and $\left\{\lambda_{n}\right\},\left\{\mu_{n}\right\} \subset(0, \infty)$ satisfy the conditions such that

$$
0 \leq \alpha_{n} \leq a<1, \quad 0<b \leq \mu_{n}, \quad \text { and } \quad 0<c \leq \lambda_{n}\|T\|^{2} \leq d<2
$$

for some $a, b, c, d \in \mathbb{R}$. Then $\left\{x_{n}\right\}$ converges strongly to $z_{0} \in A^{-1} 0 \cap T^{-1}\left(B^{-1} 0\right)$, where $z_{0}=$ $P_{A^{-1} 0 \cap T^{-1}\left(B^{-1} 0\right)^{x}}$.

\section{Proof Since}

$$
\left\|y_{n}-z\right\|^{2} \leq\left\|x_{n}-z\right\|^{2} \quad \Leftrightarrow \quad\left\|y_{n}\right\|^{2}-\left\|x_{n}\right\|^{2}-2\left\langle y_{n}-x_{n}, z\right\rangle \leq 0,
$$

it follows that $C_{n}$ is closed and convex for all $n \in \mathbb{N}$. It is obvious that $D_{n}$ is closed and convex. Then $C_{n} \cap D_{n}$ is closed and convex for all $n \in \mathbb{N}$. Let us show that $A^{-1} 0 \cap T^{-1}\left(B^{-1} 0\right) \subset$ $C_{n}$ for all $n \in \mathbb{N}$. Let $z \in A^{-1} 0 \cap T^{-1}\left(B^{-1} 0\right)$. Then $z=J_{\lambda_{n}} z$ and $T z=Q_{\mu_{n}} T z$. Since $J_{\lambda_{n}}$ is nonexpansive, we see that, for $z \in A^{-1} 0 \cap T^{-1}\left(B^{-1} 0\right)$,

$$
\begin{aligned}
\left\|z_{n}-z\right\|^{2}= & \left\|J_{\lambda_{n}}\left(x_{n}-\lambda_{n} T^{*} J_{F}\left(T x_{n}-Q_{\mu_{n}} T x_{n}\right)\right)-J_{\lambda_{n}} z\right\|^{2} \\
\leq & \left\|x_{n}-\lambda_{n} T^{*} J_{F}\left(T x_{n}-Q_{\mu_{n}} T x_{n}\right)-z\right\|^{2} \\
= & \left\|x_{n}-z-\lambda_{n} T^{*} J_{F}\left(T x_{n}-Q_{\mu_{n}} T x_{n}\right)\right\|^{2} \\
= & \left\|x_{n}-z\right\|^{2}-2\left\langle x_{n}-z, \lambda_{n} T^{*} J_{F}\left(T x_{n}-Q_{\mu_{n}} T x_{n}\right)\right\rangle \\
& +\left\|\lambda_{n} T^{*} J_{F}\left(T x_{n}-Q_{\mu_{n}} T x_{n}\right)\right\|^{2} \\
\leq & \left\|x_{n}-z\right\|^{2}-2 \lambda_{n}\left\langle T x_{n}-T z, J_{F}\left(T x_{n}-Q_{\mu_{n}} T x_{n}\right)\right\rangle \\
& +\lambda_{n}^{2}\|T\|^{2}\left\|J_{F}\left(T x_{n}-Q_{\mu_{n}} T x_{n}\right)\right\|^{2} \\
= & \left\|x_{n}-z\right\|^{2}+\lambda_{n}^{2}\|T\|^{2}\left\|T x_{n}-Q_{\mu_{n}} T x_{n}\right\|^{2} \\
& -2 \lambda_{n}\left\langle T x_{n}-Q_{\mu_{n}} T x_{n}+Q_{\mu_{n}} T x_{n}-T z, J_{F}\left(T x_{n}-Q_{\mu_{n}} T x_{n}\right)\right\rangle \\
= & \left\|x_{n}-z\right\|^{2}-2 \lambda_{n}\left\|T x_{n}-Q_{\mu_{n}} T x_{n}\right\|^{2} \\
& -2 \lambda_{n}\left\langle Q_{\mu_{n}} T x_{n}-T z, J_{F}\left(T x_{n}-Q_{\mu_{n}} T x_{n}\right)\right\rangle \\
& +\lambda_{n}^{2}\|T\|^{2}\left\|T x_{n}-Q_{\mu_{n}} T x_{n}\right\|^{2}
\end{aligned}
$$




$$
\begin{aligned}
& \leq\left\|x_{n}-z\right\|^{2}-2 \lambda_{n}\left\|T x_{n}-Q_{\mu_{n}} T x_{n}\right\|^{2}+\lambda_{n}^{2}\|T\|^{2}\left\|T x_{n}-Q_{\mu_{n}} T x_{n}\right\|^{2} \\
& =\left\|x_{n}-z\right\|^{2}+\lambda_{n}\left(\lambda_{n}\|T\|^{2}-2\right)\left\|T x_{n}-Q_{\mu_{n}} T x_{n}\right\|^{2} \\
& \leq\left\|x_{n}-z\right\|^{2}
\end{aligned}
$$

and hence

$$
\begin{aligned}
\left\|y_{n}-z\right\| & =\left\|\alpha_{n} x_{n}+\left(1-\alpha_{n}\right) z_{n}-z\right\| \\
& \leq \alpha_{n}\left\|x_{n}-z\right\|+\left(1-\alpha_{n}\right)\left\|z_{n}-z\right\| \\
& \leq \alpha_{n}\left\|x_{n}-z\right\|+\left(1-\alpha_{n}\right)\left\|x_{n}-z\right\| \\
& \leq\left\|x_{n}-z\right\| .
\end{aligned}
$$

Therefore, $A^{-1} 0 \cap T^{-1}\left(B^{-1} 0\right) \subset C_{n}$ for all $n \in \mathbb{N}$. Let us show that $A^{-1} 0 \cap T^{-1}\left(B^{-1} 0\right) \subset D_{n}$ for all $n \in \mathbb{N}$. It is obvious that $C \cap A^{-1} D \subset D_{1}$. Suppose that $A^{-1} 0 \cap T^{-1}\left(B^{-1} 0\right) \subset D_{k}$ for some $k \in \mathbb{N}$. Then $A^{-1} 0 \cap T^{-1}\left(B^{-1} 0\right) \subset C_{k} \cap D_{k}$. From $x_{k+1}=P_{C_{k} \cap D_{k}} x_{1}$, we see that

$$
\left\langle x_{k+1}-z, x_{1}-x_{k+1}\right\rangle \geq 0, \quad \forall z \in C_{k} \cap D_{k}
$$

and hence

$$
\left\langle x_{k+1}-z, x_{1}-x_{k+1}\right\rangle \geq 0, \quad \forall z \in A^{-1} 0 \cap T^{-1}\left(B^{-1} 0\right) .
$$

Then $A^{-1} 0 \cap T^{-1}\left(B^{-1} 0\right) \subset D_{k+1}$. We have by induction $A^{-1} 0 \cap T^{-1}\left(B^{-1} 0\right) \subset D_{n}$ for all $n \in \mathbb{N}$. Thus, we see that $A^{-1} 0 \cap T^{-1}\left(B^{-1} 0\right) \subset C_{n} \cap D_{n}$ for all $n \in \mathbb{N}$. This implies that $\left\{x_{n}\right\}$ is well defined.

Since $A^{-1} 0 \cap T^{-1}\left(B^{-1} 0\right)$ is nonempty, closed, and convex, there exists $z_{1} \in A^{-1} 0 \cap$ $T^{-1}\left(B^{-1} 0\right)$ such that $z_{1}=P_{A^{-1} 0 \cap T^{-1}\left(B^{-1} 0\right)} x_{1}$. From $x_{n+1}=P_{C_{n} \cap D_{n}} x_{1}$, we see that

$$
\left\|x_{1}-x_{n+1}\right\| \leq\left\|x_{1}-y\right\|
$$

for all $y \in C_{n} \cap D_{n}$. Since $z_{1} \in A^{-1} 0 \cap T^{-1}\left(B^{-1} 0\right) \subset C_{n} \cap D_{n}$, we see that

$$
\left\|x_{1}-x_{n+1}\right\| \leq\left\|x_{1}-z_{1}\right\|
$$

This means that $\left\{x_{n}\right\}$ is bounded.

Next we show that $\lim _{n \rightarrow \infty}\left\|x_{n}-x_{n+1}\right\|=0$. From the definition of $D_{n}$, we see that $x_{n}=$ $P_{D_{n}} x_{1}$. From $x_{n+1}=P_{C_{n} \cap D_{n}} x_{1}$ we have $x_{n+1} \in D_{n}$. Thus

$$
\left\|x_{n}-x_{1}\right\| \leq\left\|x_{n+1}-x_{1}\right\|
$$

for all $n \in \mathbb{N}$. This implies that $\left\{\left\|x_{1}-x_{n}\right\|\right\}$ is bounded and nondecreasing. Then there exists the limit of $\left\{\left\|x_{1}-x_{n}\right\|\right\}$. From $x_{n+1} \in D_{n}$ we see that

$$
\left\langle x_{n}-x_{n+1}, x_{1}-x_{n}\right\rangle \geq 0 .
$$


This implies from (2.3) that

$$
0 \leq\left\|x_{n+1}-x_{1}\right\|^{2}-\left\|x_{n}-x_{1}\right\|^{2}-\left\|x_{n+1}-x_{n}\right\|^{2}
$$

and hence

$$
\left\|x_{n+1}-x_{n}\right\|^{2} \leq\left\|x_{n+1}-x_{1}\right\|^{2}-\left\|x_{n}-x_{1}\right\|^{2} .
$$

Since there exists the limit of $\left\{\left\|x_{1}-x_{n}\right\|\right\}$, we see that

$$
\lim _{n \rightarrow \infty}\left\|x_{n}-x_{n+1}\right\|=0
$$

From $x_{n+1} \in C_{n}$, we also see that $\left\|y_{n}-x_{n+1}\right\| \leq\left\|x_{n}-x_{n+1}\right\|$. Then we get from (3.3) that $\left\|y_{n}-x_{n+1}\right\| \rightarrow 0$. Using this, we have

$$
\left\|y_{n}-x_{n}\right\| \leq\left\|y_{n}-x_{n+1}\right\|+\left\|x_{n+1}-x_{n}\right\| \rightarrow 0 \text {. }
$$

We have from (3.1), for any $z \in A^{-1} 0 \cap T^{-1}\left(B^{-1} 0\right)$,

$$
\begin{aligned}
\left\|y_{n}-z\right\|^{2}= & \left\|\alpha_{n} x_{n}+\left(1-\alpha_{n}\right) z_{n}-z\right\|^{2} \\
\leq & \alpha_{n}\left\|x_{n}-z\right\|^{2}+\left(1-\alpha_{n}\right)\left\|z_{n}-z\right\|^{2} \\
\leq & \alpha_{n}\left\|x_{n}-z\right\|^{2}+\left(1-\alpha_{n}\right)\left\|x_{n}-z\right\|^{2} \\
& +\left(1-\alpha_{n}\right) \lambda_{n}\left(\lambda_{n}\|T\|^{2}-2\right)\left\|T x_{n}-Q_{\mu_{n}} T x_{n}\right\|^{2} \\
\leq & \left\|x_{n}-z\right\|^{2}+\left(1-\alpha_{n}\right) \lambda_{n}\left(\lambda_{n}\|T\|^{2}-2\right)\left\|T x_{n}-Q_{\mu_{n}} T x_{n}\right\|^{2} .
\end{aligned}
$$

Thus we see that

$$
\begin{aligned}
(1 & \left.-\alpha_{n}\right) \lambda_{n}\left(2-\lambda_{n}\|T\|^{2}\right)\left\|T x_{n}-Q_{\mu_{n}} T x_{n}\right\|^{2} \\
& \leq\left\|x_{n}-z\right\|^{2}-\left\|y_{n}-z\right\|^{2} \\
& =\left(\left\|x_{n}-z\right\|+\left\|y_{n}-z\right\|\right)\left(\left\|x_{n}-z\right\|-\left\|y_{n}-z\right\|\right) \\
& \leq\left(\left\|x_{n}-z\right\|+\left\|y_{n}-z\right\|\right)\left\|x_{n}-y_{n}\right\| .
\end{aligned}
$$

From $\left\|y_{n}-x_{n}\right\| \rightarrow 0,0 \leq \alpha_{n} \leq a<1$, and $0<c \leq \lambda_{n}\|T\|^{2} \leq d<2$, we see that

$$
\lim _{n \rightarrow \infty}\left\|T x_{n}-Q_{\mu_{n}} T x_{n}\right\|^{2}=0
$$

We also see that $\left\|y_{n}-x_{n}\right\|=\left\|\alpha_{n} x_{n}+\left(1-\alpha_{n}\right) z_{n}-x_{n}\right\|=\left(1-\alpha_{n}\right)\left\|z_{n}-x_{n}\right\|$. From $\left\|y_{n}-x_{n}\right\| \rightarrow 0$ and $0 \leq \alpha_{n} \leq a<1$, we see that

$$
\lim _{n \rightarrow \infty}\left\|x_{n}-z_{n}\right\|=0
$$

Since $\left\{x_{n}\right\}$ is bounded, there exists a subsequence $\left\{x_{n_{i}}\right\}$ of $\left\{x_{n}\right\}$ converging weakly to $w$. From (3.4) $\left\{y_{n_{i}}\right\}$ converges weakly to $w$. Furthermore, from (3.6) $\left\{z_{n_{i}}\right\}$ converges weakly to $w$. Since $T$ is bounded and linear, we also see that $\left\{T x_{n_{i}}\right\}$ converges weakly to $T w$. Using this and $\lim _{n \rightarrow \infty}\left\|T x_{n}-Q_{\mu_{n}} T x_{n}\right\|=0$, we see that $Q_{\mu_{n}} T x_{n_{i}} \rightarrow T w$. Since $Q_{\mu_{n}}$ is the metric 
resolvent of $B$ for $\mu_{n}>0$, we see that $\frac{J_{F}\left(T x_{n}-Q_{\mu_{n}} T x_{n}\right)}{\mu_{n}} \in B Q_{\mu_{n}} T x_{n}$ for all $n \in \mathbb{N}$. From the monotonicity of $B$ we see that

$$
0 \leq\left\langle u-Q_{\mu_{n_{i}}} T x_{n_{i}}, v^{*}-\frac{J_{F}\left(T x_{n_{i}}-Q_{\mu_{n_{i}}} T x_{n_{i}}\right)}{\mu_{n_{i}}}\right\rangle
$$

for all $\left(u, v^{*}\right) \in B$. Taking $i \rightarrow \infty$, we have, from $\left\|J_{F}\left(T x_{n_{i}}-Q_{\mu_{n_{i}}} T x_{n_{i}}\right)\right\|=\| T x_{n_{i}}-$ $Q_{\mu_{n_{i}}} T x_{n_{i}} \| \rightarrow 0$ and $0<b \leq \mu_{n_{i}}, 0 \leq\left\langle u-T w, v^{*}-0\right\rangle$ for all $\left(u, v^{*}\right) \in B$. Since $B$ is maximal monotone, we see that $T w \in B^{-1} 0$. This implies that $w \in T^{-1}\left(B^{-1} 0\right)$. Since $z_{n}=$ $J_{\lambda_{n}}\left(x_{n}-\lambda_{n} T^{*} J_{F}\left(T x_{n}-Q_{\mu_{n}} T x_{n}\right)\right)$, we see that

$$
\begin{aligned}
z_{n} & =J_{\lambda_{n}}\left(x_{n}-\lambda_{n} T^{*} J_{F}\left(T x_{n}-Q_{\mu_{n}} T x_{n}\right)\right) \\
& \Leftrightarrow x_{n}-\lambda_{n} T^{*} J_{F}\left(T x_{n}-Q_{\mu_{n}} T x_{n}\right) \in z_{n}+\lambda_{n} A z_{n} \\
& \Leftrightarrow \quad x_{n}-z_{n}-\lambda_{n} T^{*} J_{F}\left(T x_{n}-Q_{\mu_{n}} T x_{n}\right) \in \lambda_{n} A z_{n} \\
& \Leftrightarrow \frac{1}{\lambda_{n}}\left(x_{n}-z_{n}-\lambda_{n} T^{*} J_{F}\left(T x_{n}-Q_{\mu_{n}} T x_{n}\right)\right) \in A z_{n} .
\end{aligned}
$$

Since $A$ is monotone, we see that, for $(u, v) \in A$,

$$
\left\langle z_{n}-u, \frac{1}{\lambda_{n}}\left(x_{n}-z_{n}-\lambda_{n} T^{*} J_{F}\left(T x_{n}-Q_{\mu_{n}} T x_{n}\right)\right)-v\right\rangle \geq 0
$$

and hence

$$
\left\langle z_{n}-u, \frac{x_{n}-z_{n}}{\lambda_{n}}-T^{*} J_{F}\left(T x_{n}-Q_{\mu_{n}} T x_{n}\right)-v\right\rangle \geq 0 .
$$

Replacing $n$ by $n_{i}$, we see that

$$
\left\langle z_{n_{i}}-u, \frac{x_{n_{i}}-z_{n_{i}}}{\lambda_{n_{i}}}-T^{*} J_{F}\left(T x_{n_{i}}-Q_{\mu_{n_{i}}} T x_{n_{i}}\right)-v\right\rangle \geq 0 .
$$

Since $x_{n_{i}}-z_{n_{i}} \rightarrow 0,0<c \leq \lambda_{n_{i}}\|T\|^{2}, z_{n_{i}} \rightarrow w$ and $T^{*} J_{F}\left(T x_{n}-Q_{\mu_{n_{i}}} T x_{n_{i}}\right) \rightarrow 0$, we see that $\langle w-u,-v\rangle \geq 0$. Since $A$ is maximal monotone, we see that $0 \in A w$. Therefore, $w \in A^{-1} 0 \cap$ $T^{-1}\left(B^{-1} 0\right)$.

From $z_{1}=P_{A^{-1} 0 \cap T^{-1}\left(B^{-1} 0\right)}^{x_{1}}, w \in A^{-1} 0 \cap T^{-1}\left(B^{-1} 0\right)$ and (3.2), we see that

$$
\begin{aligned}
\left\|x_{1}-z_{1}\right\| & \leq\left\|x_{1}-w\right\| \leq \liminf _{i \rightarrow \infty}\left\|x_{1}-x_{n_{i}}\right\| \\
& \leq \limsup _{i \rightarrow \infty}\left\|x_{1}-x_{n_{i}}\right\| \leq\left\|x_{1}-z_{1}\right\| .
\end{aligned}
$$

Then we get

$$
\lim _{i \rightarrow \infty}\left\|x_{1}-x_{n_{i}}\right\|=\left\|x_{1}-w\right\|=\left\|x_{1}-z_{1}\right\| .
$$

Since $H$ satisfies the Kadec-Klee property, we see that $x_{1}-x_{n_{i}} \rightarrow x_{1}-w$ and hence $x_{n_{i}} \rightarrow$ $w=z_{1}$. Therefore, we have $x_{n} \rightarrow w=z_{1}$. This completes the proof.

Next, using the shrinking projection method introduced by Takahashi et al. [13], we prove a strong convergence theorem for finding a solution of the split common null point 
problem in Banach spaces. Before proving the theorem, we need the following lemma, which was proved by Takahashi [24].

Lemma 10 Let $E$ and $F$ be uniformly convex and smooth Banach spaces and let $J_{E}$ and $J_{F}$ be the duality mappings on $E$ and $F$, respectively. Let $A$ and $B$ be maximal monotone operators of $E$ into $2^{E^{*}}$ and $F$ into $2^{F^{*}}$ such that $A^{-1} 0 \neq \emptyset$ and $B^{-1} 0 \neq \emptyset$, respectively. Let $J_{\lambda}$ and $Q_{\mu}$ be the metric resolvents of $A$ for $\lambda>0$ and $B$ for $\mu>0$, respectively. Let $T: E \rightarrow F$ be a bounded linear operator such that $T \neq 0$ and let $T^{*}$ be the adjoint operator of $T$. Suppose that $A^{-1} 0 \cap T^{-1}\left(B^{-1} 0\right) \neq \emptyset$. Let $\lambda, \mu, r>0$ and $z \in E$. Then the following are equivalent:

(i) $z=J_{\lambda}\left(z-r J_{E}^{-1} T^{*} J_{F}\left(T z-Q_{\mu} T z\right)\right)$;

(ii) $z \in A^{-1} 0 \cap T^{-1}\left(B^{-1} 0\right)$.

Theorem 11 Let $H$ be a Hilbert space and let $F$ be a uniformly convex Banach space whose norm is Fréchet differentiable. Let $J_{F}$ be the duality mapping on $F$. Let $A$ and $B$ be maximal monotone operators of $H$ into $2^{H}$ and $F$ into $2^{F^{*}}$ such that $A^{-1} 0 \neq \emptyset$ and $B^{-1} 0 \neq \emptyset$, respectively. Let $J_{\lambda}$ be the resolvent of $A$ for $\lambda>0$ and let $Q_{\mu}$ be the metric resolvent of $B$ for $\mu>0$. Let $T: H \rightarrow F$ be a bounded linear operator such that $T \neq 0$ and let $T^{*}$ be the adjoint operator of $T$. Suppose that $A^{-1} 0 \cap T^{-1}\left(B^{-1} 0\right) \neq \emptyset$. Let $\left\{u_{n}\right\}$ be a sequence in $H$ such that $u_{n} \rightarrow u$. Let $x_{1} \in H, C_{1}=H$, and $\left\{x_{n}\right\}$ be a sequence generated by

$$
\left\{\begin{array}{l}
z_{n}=J_{\lambda_{n}}\left(x_{n}-\lambda_{n} T^{*} J_{F}\left(T x_{n}-Q_{\mu} T x_{n}\right)\right), \\
C_{n+1}=\left\{z \in H:\left\|z_{n}-z\right\| \leq\left\|x_{n}-z\right\|\right\} \cap C_{n}, \\
x_{n+1}=P_{C_{n+1}} u_{n+1}, \quad \forall n \in \mathbb{N},
\end{array}\right.
$$

where $0<c \leq \lambda_{n}\|T\|^{2} \leq 2$ and $0<\mu$ for some $c \in \mathbb{R}$. Then $\left\{x_{n}\right\}$ converges strongly to a point $z_{0} \in A^{-1} 0 \cap T^{-1}\left(B^{-1} 0\right)$, where $z_{0}=P_{A^{-1} 0 \cap T^{-1}\left(B^{-1} 0\right)} u$.

Proof We first show that the sequence $\left\{x_{n}\right\}$ is well defined. Let $x_{1} \in H$ and $z_{n}=J_{\lambda_{n}}\left(x_{n}-\right.$ $\left.\lambda_{n} T^{*} J_{F}\left(T x_{n}-Q_{\mu} T x_{n}\right)\right)$ with $0<c \leq \lambda_{n}\|T\|^{2} \leq 2$. As in the proof of Theorem 9, we see that, for $z \in A^{-1} 0 \cap T^{-1}\left(B^{-1} 0\right)$,

$$
\begin{aligned}
\left\|z_{n}-z\right\|^{2}= & \left\|J_{\lambda_{n}}\left(x_{n}-\lambda_{n} T^{*} J_{F}\left(T x_{n}-Q_{\mu} T x_{n}\right)\right)-J_{\lambda_{n}} z\right\|^{2} \\
\leq & \left\|x_{n}-\lambda_{n} T^{*} J_{F}\left(T x_{n}-Q_{\mu} T x_{n}\right)-z\right\|^{2} \\
\leq & \left\|x_{n}-z\right\|^{2}-2 \lambda_{n}\left\|T x_{n}-Q_{\mu} T x_{n}\right\|^{2} \\
& -2 \lambda_{n}\left\langle Q_{\mu} T x_{n}-T z, J_{F}\left(T x_{n}-Q_{\mu} T x_{n}\right)\right\rangle+\lambda_{n}^{2}\|T\|^{2}\left\|T x_{n}-Q_{\mu} T x_{n}\right\|^{2} \\
\leq & \left\|x_{n}-z\right\|^{2}-2 \lambda_{n}\left\|T x_{n}-Q_{\mu} T x_{n}\right\|^{2}+\lambda_{n}^{2}\|T\|^{2}\left\|T x_{n}-Q_{\mu} T x_{n}\right\|^{2} \\
= & \left\|x_{n}-z\right\|^{2}+\lambda_{n}\left(\lambda_{n}\|T\|^{2}-2\right)\left\|T x_{n}-Q_{\mu} T x_{n}\right\|^{2} \\
\leq & \left\|x_{n}-z\right\|^{2} .
\end{aligned}
$$

Therefore, $A^{-1} 0 \cap T^{-1}\left(B^{-1} 0\right) \subset C_{n}$ for all $n \in \mathbb{N}$. Moreover, since

$$
\begin{aligned}
\left\{z \in H:\left\|z_{n}-z\right\| \leq\left\|x_{n}-z\right\|\right\} & =\left\{z \in H:\left\|z_{n}-z\right\|^{2} \leq\left\|x_{n}-z\right\|^{2}\right\} \\
& =\left\{z \in H:\left\|z_{n}\right\|^{2}-\left\|x_{n}\right\|^{2} \leq 2\left\langle z_{n}-x_{n}, z\right\rangle\right\}
\end{aligned}
$$


it is closed and convex. Applying these facts inductively, we find that $C_{n}$ is nonempty, closed, and convex for every $n \in \mathbb{N}$, and hence $\left\{x_{n}\right\}$ is well defined.

Let $C_{0}=\bigcap_{n=1}^{\infty} C_{n}$. Then since $C_{0} \supset A^{-1} 0 \cap T^{-1}\left(B^{-1} 0\right) \neq \emptyset, C_{0}$ is also nonempty. Let $w_{n}=P_{C_{n}} u$ for every $n \in \mathbb{N}$. Then, by Lemma 8 , we have $w_{n} \rightarrow z_{0}=P_{C_{0}} u$. Since a metric projection on $H$ is nonexpansive, it follows that

$$
\begin{aligned}
\left\|x_{n}-z_{0}\right\| & \leq\left\|x_{n}-w_{n}\right\|+\left\|w_{n}-z_{0}\right\| \\
& =\left\|P_{C_{n}} u_{n}-P_{C_{n}} u\right\|+\left\|w_{n}-z_{0}\right\| \\
& \leq\left\|u_{n}-u\right\|+\left\|w_{n}-z_{0}\right\|
\end{aligned}
$$

and hence $x_{n} \rightarrow z_{0}$.

Since $z_{0} \in C_{0} \subset C_{n+1}$, we have $\left\|z_{n}-z_{0}\right\| \leq\left\|x_{n}-z_{0}\right\|$ for all $n \in \mathbb{N}$. Letting $n \rightarrow \infty$, we get $z_{n} \rightarrow z_{0}$. By the assumption of $\left\{\lambda_{n}\right\}$, there exists a subsequence $\left\{\lambda_{n_{i}}\right\}$ of $\left\{\lambda_{n}\right\}$ converging to $\lambda_{0}$. From $0<c \leq \lambda_{n}\|T\|^{2} \leq 2$, we see that $0<c \leq \lambda_{0}\|T\|^{2} \leq 2$. Put $v_{n}=x_{n}-J_{\lambda_{n}} T^{*} J_{F}\left(T x_{n}-\right.$ $\left.Q_{\mu} T x_{n}\right)$. We see that

$$
\begin{aligned}
\left\|J_{\lambda_{0}}\left(I-\lambda_{0} T^{*} J_{F}\left(T-Q_{\mu} T\right)\right) x_{n_{i}}-z_{n_{i}}\right\| \\
=\left\|J_{\lambda_{0}}\left(I-\lambda_{0} T^{*} J_{F}\left(T-Q_{\mu} T\right)\right) x_{n_{i}}-J_{\lambda_{n_{i}}}\left(I-\lambda_{n_{i}} T^{*} J_{F}\left(T-Q_{\mu} T\right)\right) x_{n_{i}}\right\| \\
=\| J_{\lambda_{0}}\left(I-\lambda_{0} T^{*} J_{F}\left(T-Q_{\mu} T\right)\right) x_{n_{i}}-J_{\lambda_{0}}\left(I-\lambda_{n_{i}} T^{*} J_{F}\left(T-Q_{\mu} T\right)\right) x_{n_{i}} \\
\quad+J_{\lambda_{0}}\left(I-\lambda_{n_{i}} T^{*} J_{F}\left(T-Q_{\mu} T\right)\right) x_{n_{i}}-J_{\lambda_{n_{i}}}\left(I-\lambda_{n_{i}} T^{*} J_{F}\left(T-Q_{\mu} T\right)\right) x_{n_{i}} \| \\
\leq\left\|\left(I-\lambda_{0} T^{*} J_{F}\left(T-Q_{\mu} T\right)\right) x_{n_{i}}-\left(I-\lambda_{n_{i}} T^{*} J_{F}\left(T-Q_{\mu} T\right)\right) x_{n_{i}}\right\| \\
\quad+\left\|J_{\lambda_{0}} v_{n_{i}}-J_{\lambda_{n_{i}}} v_{n_{i}}\right\| \\
\leq\left|\lambda_{0}-\lambda_{n_{i}}\right|\left\|T^{*} J_{F}\left(T-Q_{\mu} T\right) x_{n_{i}}\right\|+\frac{\left|\lambda_{0}-\lambda_{n_{i}}\right|}{\lambda_{0}}\left\|J_{\lambda_{0}} v_{n_{i}}-v_{n_{i}}\right\| \rightarrow 0 .
\end{aligned}
$$

On the other hand, since $J_{\lambda_{0}}, T^{*}, Q_{\mu}$, and $T$ are all continuous, $J_{\lambda_{0}}\left(I-\lambda_{0} T^{*} J_{F}\left(T-Q_{\mu} T\right)\right)$ is continuous. Then we see that

$$
\left\|J_{\lambda_{0}}\left(I-\lambda_{0} T^{*} J_{F}\left(T-Q_{\mu} T\right)\right) x_{n_{i}}-J_{\lambda_{0}}\left(I-\lambda_{0} T^{*} J_{F}\left(T-Q_{\mu} T\right)\right) z_{0}\right\| \rightarrow 0
$$

Hence we see that

$$
\begin{aligned}
\| z_{0} & -J_{\lambda_{0}}\left(I-\lambda_{0} T^{*} J_{F}\left(T-Q_{\mu} T\right)\right) z_{0} \| \\
\leq & \left\|z_{0}-z_{n_{i}}\right\|+\left\|z_{n_{i}}-J_{\lambda_{0}}\left(I-\lambda_{0} T^{*} J_{F}\left(T-Q_{\mu} T\right)\right) x_{n_{i}}\right\| \\
& \quad+\left\|J_{\lambda_{0}}\left(I-\lambda_{0} T^{*} J_{F}\left(T-Q_{\mu} T\right)\right) x_{n_{i}}-J_{\lambda_{0}}\left(I-\lambda_{0} T^{*} J_{F}\left(T-Q_{\mu} T\right)\right) z_{0}\right\| \\
& \rightarrow 0 .
\end{aligned}
$$

This implies $z_{0} \in A^{-1} 0 \cap T^{-1}\left(B^{-1} 0\right)$ by Lemma 10 . Since $z_{0}=P_{C_{0}} u \in A^{-1} 0 \cap T^{-1}\left(B^{-1} 0\right)$ and $A^{-1} 0 \cap T^{-1}\left(B^{-1} 0\right) \subset C_{0}$, we have $z_{0}=P_{A^{-1} 0 \cap T^{-1}\left(B^{-1} 0\right)} u$, which completes the proof.

We do not know whether the Hilbert space $H$ in Theorems 9 and 11 can be replaced by a Banach space $E$. 


\section{Competing interests}

The authors declare that they have no competing interests.

\section{Authors' contributions}

All authors contributed equally and significantly in writing this article. All authors read and approved the final manuscript.

\section{Author details}

${ }^{1}$ Center for Fundamental Science, Kaohsiung Medical University, Kaohsiung, 80702, Taiwan. ${ }^{2}$ Keio Research and Education Center for Natural Sciences, Keio University, Kouhoku-ku, Yokohama, 223-8521, Japan. ${ }^{3}$ Department of Mathematical and Computing Sciences, Tokyo Institute of Technology, Ookayama, Meguro-ku, Tokyo, 152-8552, Japan. ${ }^{4}$ Center for General Education, China Medical University, Taichung, 40402, Taiwan. ${ }^{5}$ Department of Mathematics, King Abdulaziz University, P.O. Box 80203, Jeddah, 21589, Saudi Arabia.

\section{Acknowledgements}

The first author was partially supported by Grant-in-Aid for Scientific Research No. 15K04906 from Japan Society for the Promotion of Science. The second author was partially supported by the grant MOST 102-2115-M-039-003-MY3.

\section{Received: 20 March 2015 Accepted: 11 May 2015 Published online: 14 June 2015}

\section{References}

1. Censor, Y, Elfving, T: A multiprojection algorithm using Bregman projections in a product space. Numer. Algorithms 8 , 221-239 (1994)

2. Alsulami, SM, Takahashi, W: The split common null point problem for maximal monotone mappings in Hilbert spaces and applications. J. Nonlinear Convex Anal. 15, 793-808 (2014)

3. Byrne, C, Censor, Y, Gibali, A, Reich, S: The split common null point problem. J. Nonlinear Convex Anal. 13, 759-775 (2012)

4. Censor, Y, Segal, A: The split common fixed-point problem for directed operators. J. Convex Anal. 16, $587-600$ (2009)

5. Moudafi, A: The split common fixed point problem for demicontractive mappings. Inverse Probl. 26, 055007 (2010) 6 pp.

6. Takahashi, W, Xu, H-K, Yao, J-C: Iterative methods for generalized split feasibility problems in Hilbert spaces. Set-Valued Var. Anal. 23, 205-221 (2015)

7. Takahashi, W: The split feasibility problem in Banach spaces. J. Nonlinear Convex Anal. 15, 1349-1355 (2014)

8. Takahashi, W: The split feasibility problem and the shrinking projection method in Banach spaces. J. Nonlinear Convex Anal. (to appear)

9. Nakajo, K, Takahashi, W: Strong convergence theorems for nonexpansive mappings and nonexpansive semigroups. J. Math. Anal. Appl. 279, 372-379 (2003)

10. Alsulami, SM, Latif, A, Takahashi, W: Strong convergence theorems by hybrid methods for the split feasibility problem in Banach spaces. Linear Nonlinear Anal. (to appear)

11. Ohsawa, S, Takahashi, W: Strong convergence theorems for resolvents of maximal monotone operators in Banach spaces. Arch. Math. (Basel) 81, 439-445 (2003)

12. Solodov, MV, Svaiter, BF: Forcing strong convergence of proximal point iterations in a Hilbert space. Math. Program. Ser. A 87, 189-202 (2000)

13. Takahashi, W, Takeuchi, Y, Kubota, R: Strong convergence theorems by hybrid methods for families of nonexpansive mappings in Hilbert spaces. J. Math. Anal. Appl. 341, 276-286 (2008)

14. Takahashi, W: Introduction to Nonlinear and Convex Analysis. Yokohama Publishers, Yokohama (2009)

15. Takahashi, W: Nonlinear Functional Analysis. Yokohama Publishers, Yokohama (2000)

16. Takahashi, W: Convex Analysis and Approximation of Fixed Points. Yokohama Publishers, Yokohama (2000) (Japanese)

17. Kamimura, S, Takahashi, W: Strong convergence of proximal-type algorithm in a Banach space. SIAM J. Optim. 13, 938-945 (2002)

18. Browder, FE: Nonlinear maximal monotone operators in Banach spaces. Math. Ann. 175, 89-113 (1968)

19. Aoyama, K, Kohsaka, F, Takahashi, W: Three generalizations of firmly nonexpansive mappings: their relations and continuous properties. J. Nonlinear Convex Anal. 10, 131-147 (2009)

20. Eshita, K, Takahashi, W: Approximating zero points of accretive operators in general Banach spaces. JP J. Fixed Point Theory Appl. 2, 105-116 (2007)

21. Takahashi, S, Takahashi, W, Toyoda, M: Strong convergence theorems for maximal monotone operators with nonlinear mappings in Hilbert spaces. J. Optim. Theory Appl. 147, 27-41 (2010)

22. Mosco, U: Convergence of convex sets and of solutions of variational inequalities. Adv. Math. 3, $510-585$ (1969)

23. Tsukada, M: Convergence of best approximations in a smooth Banach space. J. Approx. Theory 40, 301-309 (1984)

24. Takahashi, W: The split common null point problem in Banach spaces. Arch. Math. (Basel) 104, 357-365 (2015) 